\title{
Teaching number skills and concepts with Stern Structural Arithmetic materials
}

\section{Vikki Horner}

This paper discusses the use of Stern teaching materials with children with Down syndrome. The theory underlying the design of the materials is discussed, the teaching approach and methodology are described and evidence supporting effectiveness is outlined.

Stern Structural Arithmetic

A programme for learning number concepts that is based on reasoning and insight into mathematical relationships, rather than rote learning and counting.

Primary Framework Part of a UK national strategy to help practitioners, teachers, schools and settings increase all children's access to excellent teaching. The electronic Framework can be accessed at www.standards.dfes.gov.uk/ primaryFramework.
Maths Extra Limited, 3 North Street, Mere, Wiltshire, BA12 $6 \mathrm{HH}, \mathrm{UK}$

Correspondence to: Vikki Horner•e-mail:vikki.horner@ mathsextra.com

doi:10.3104/practice.2020

(๑) 2007 The Authors. Journal Compilation @ 2007 The Down Syndrome Educational Trust.

\section{The theory}

The Structural Arithmetic materials were designed to enable children to learn from their own discoveries thus gaining insight into the number system. The multi-sensory apparatus was specifically designed to make the structure of the number system visible. Children work with two different sets of representations of numbers 1 to 10 in the form of blocks of $2 \mathrm{~cm}$ cubes of varying lengths, the size being relevant to the child's sensori-motor development, and a set of pattern boards providing a specific series of structured patterns (first advocated by Dr Catherine Stern) based on the characteristics of odd and even numbers. The self-checking aspects of the materials have two functions; through experimentation with the materials children 'see' and feel' in which way a block does not fit and try others until they are successful; they are excellent diagnostic tools for assessment. One of the most important principles built into the Stern materials is in their arrangement as it focuses attention on the relationships between numbers, (a requirement of the renewed 'Primary Framework' for maths in England and Wales). When using the apparatus multiple relationships are in view and in the child's mind at the same time.

For children with learning difficulties/disabilities, these materials are of special importance. They may have difficulties with discrimination, memory, inter-sensory organisation, perceptual processing (both visual and auditory) and difficulties in sustaining their attention. In order to form concepts these children must learn how to receive and integrate information from as many different senses as possible. Structural Arithmetic allows children's thinking to grow out of their own experimentation with the materials. Daily use is recommended. However, very positive progress has been achieved by using the programme three times a week (see Evidence, below).

\section{Visualisation}

It is generally understood that children use mental pictures to support their thinking. Stern's apparatus is specifically designed to develop a child's ability to form images. However, it is not sufficient for children to picture an image in isolation, they must be able to visualise it in relation to other numbers and to visualise the actions that can be performed with it.

\section{Language}

Children can be helped to understand the meaning of spoken language by letting them see and touch what the words mean. For example "What number comes between 4 and 6?" A child finds the 4-block in the Counting Board and places her hand over it, then finds the 6-block and covers it with the other hand, immediately the child gets a physical experience, and a clear image describing the meaning of the word 'between'. Words not understood are easily forgotten.

\section{Receptive language, auditory memory}

In carrying out spoken instructions, children develop their skills in comprehending receptive language and in verbal memory. It is fun for them to follow directions as part of a game. For example, scatter blocks 1 to 10 on a table nearby and ask a child to go and get the 6-block and the block one smaller than 6 . 


\section{Action}

The Structural Arithmetic materials enable children to perform actions at every step which helps them to develop mathematical concepts. For example the teacher displays a pattern of 4 cubes and asks the children to name the amount. Then she hides the pattern behind a screen and 'does something to it'. Next she reveals a change of pattern (now 2 cubes) and asks, "What did I do to it?" How many are left?" After studying the new pattern they answer "You took 2 cubes away, 2 are left." To say this, they must have been able to reconstruct in their minds what took place behind the screen - the subtraction of 2 cubes. Experiments like this provide practice in visualising an action or an operation.

\section{Reversibility}

Structural Arithmetic programmes present concepts in such a way that children can always work out how they can 'undo' what has been 'done'. For example they are able to see that adding 1 cube to a pattern of 4 cubes creates the next higher number pattern. Right away they learn to reverse this. They subtract 1 cube from the 5pattern and find that it takes them right back to the original 4-pattern. In a different experiment, children learn that the 6-block and the 4-block together measure 10 . They find it entirely logical then that by removing the 6-block from this combination, the 4-block must remain. From the beginning they understand that subtraction 'undoes' what adding has 'done' realising that addition and subtraction have a special relationship to each other.

\section{Assessment tools}

The materials can be useful for assessing the following abilities: to visualise, to comprehend spoken language, to put ideas into words, to remember names, to describe a category, to learn from sensori-motor experiences, to turn concepts around in the mind, to remember symbols, to reason and make inferences, and to develop the coordination required for recording numbers and equations.

\section{Cognitive growth}

Hand-eye coordination - improves as children practise over and over again to fit blocks into matching grooves. The ability to scan - develops as children search for one block among many scattered blocks. The ability to judge sizes - is developed when children constantly compare blocks with empty grooves to find a matching combination. Left/right directionality and one-to-one correspondence - is practised as children fit cubes into the empty pattern boards. Spatial awareness - is increased throughout the programme.

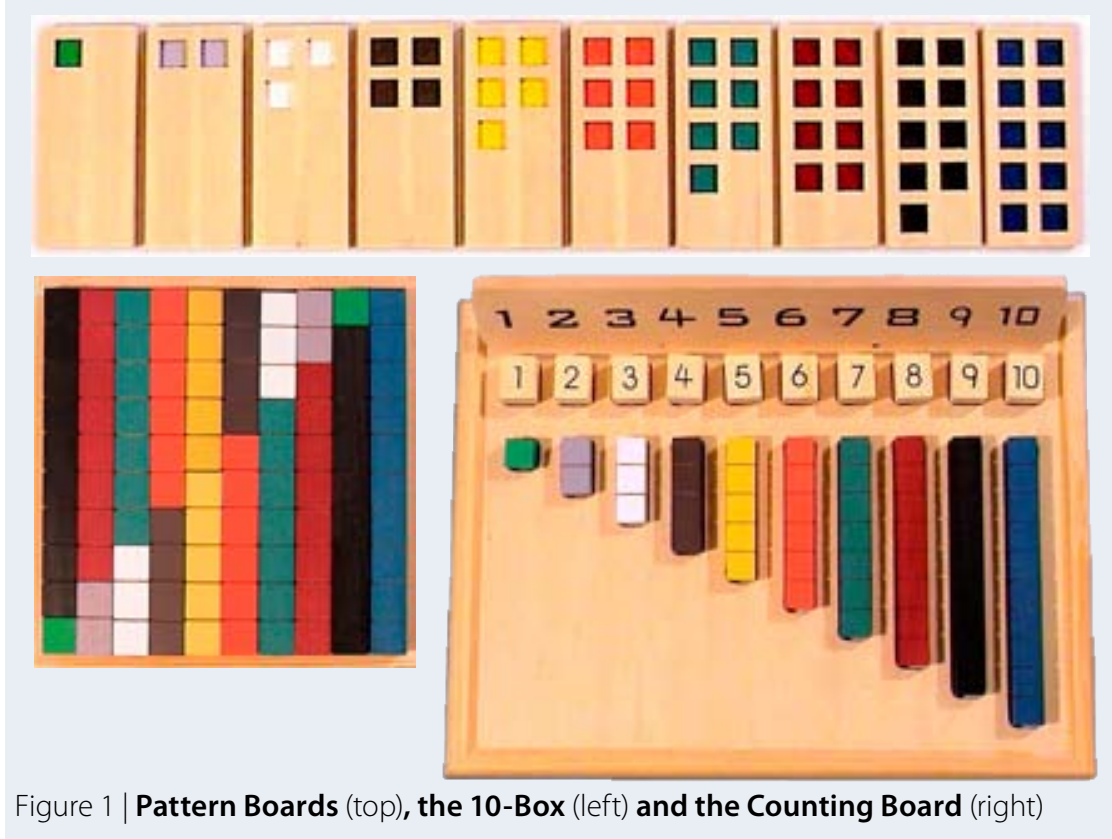

\section{The approach}

\section{Kit A}

Experimenting with Numbers (number facts to 10) -

Children learn the basic addition and subtraction facts with numbers up to and including 10. Taught in small-steps, through three stages, each level broadens previous learning.

Beginning at level 1, simple experiments take place in the Counting Board (CB); 10-Box (TB), and Pattern Boards (PB). Children discover 'size' relationships, position and sequencing (CB). Filling the 10-Box with pairs of blocks (later to become the bonds to 10), place a random block in the 10-Box; a child finds the block that fits. Find two pairs of like blocks, (preparation for the commutativity of addition) build the staircase (TB). Recognise and match patterns, construct patterns, sequence from the smallest to the biggest, to know where each pattern lives in the sequence (PB). After the 'puzzle' stage of experiments, level 2 introduces language, broadens and builds further understanding. Children are keen to talk about what has been discovered so will learn the name of each Block and Pattern Board; learn to count; know which block is meant when described with the words 'one bigger' 'one smaller' 'after' 'before' 'between' and 'equal to'; will add ' 0 ' to any number and know that it results in the same number; zero - will know that 10 and nothing makes 10; will add 1 and see that it is the next higher number; add 2 to an even number and learn that it gives the next higher even number; add 2 to an odd number gives the next highest odd number. When subtracting 1 from any number, the apparatus provides unforgettable imagery showing that this results in the 


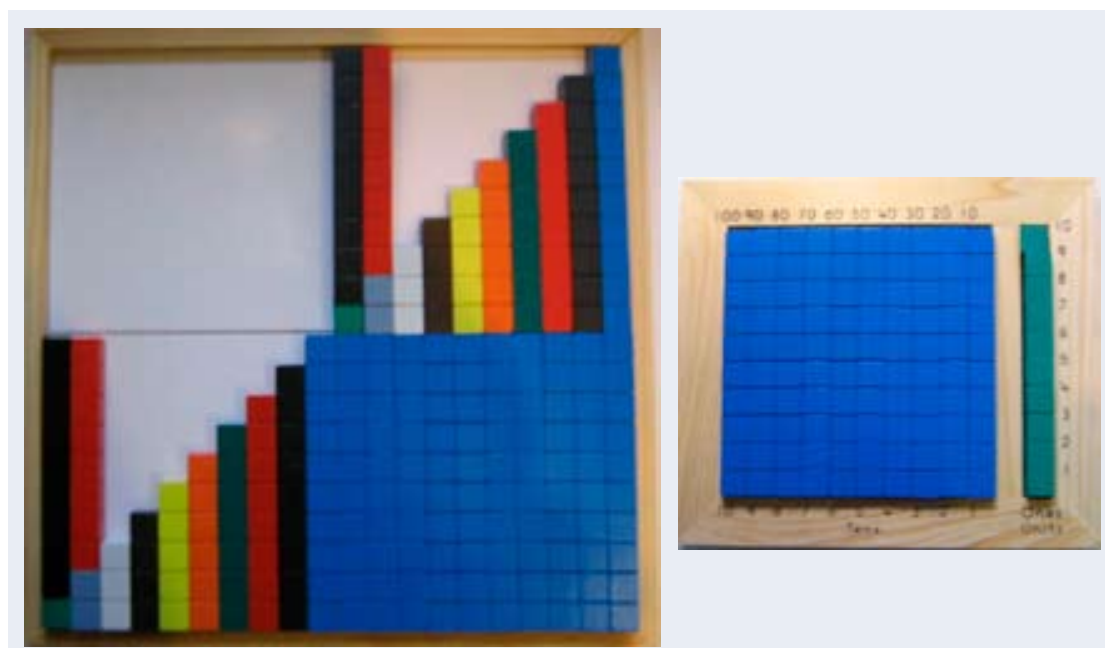

Figure 2 | The 20-Tray and the Dual Board

next lower number and subtracting 2 from an even/odd number results in the next lower even/ odd number.

At this stage in the 10-Box, with the number names in place, children can now name the familiar combinations of blocks that go together to fill the box and express them orally for example: 8 and 2 makes 10,10 and nothing makes 10 . They will become aware that the two like pairs of blocks can be placed in any order and it makes no difference to the sum. " 8 and 2 makes 10,2 and 8 makes 10 ." Here also, they will begin to understand the concept and language of the 'missing' addend, " 8 and what makes 10 ?" Hide two blocks behind your back and say "I have 10 altogether. In one hand I have 8, (show it) what is in the other hand?" Pupils begin to see the relationship between addition and subtraction that of 'doing' and 'undoing'. When your child gets to be the 'teacher' this is a wonderful way of assessing how many facts are known. Work continues with the bonds 1 - 9 by filling smaller number boxes with blocks, children work out the combinations with sums less than 10.

With this knowledge in place it is time to move to level 3 which introduces the numerals 1 to 10 and links them to the named number-blocks and Pattern Boards. Equation work begins. To do this the plus, minus and equal signs are introduced. With the pattern boards and cubes children are able to act out a subtraction word problem; able to read and understand an equation and use the plus or minus sign in an equation. Back to the 10-Box children begin to record from memory addition facts with the sum of 10 and record the related subtraction facts. Using the wooden number markers, children will record an equation from hearing an addition or a subtraction story. For children who have difficulties with handwriting, recording using number markers will not hinder progress.

\section{Kit B}

Number Bonds to 20 - Once the foundation is in place the bonds to 20 are tackled using the 20 Tray and will transfer relational understanding of the bonds to 10 . With the size of this piece of apparatus children can clearly see that the same ordered blocks 1 to 10 now sit on top of a base of ten 10-blocks. They 'see' that the same facts to 10 hold true in the structure of the teen numbers.

\section{Transferring basic facts to higher decades}

Learning about place-value using the Dual Board really makes this concept visible and enables the transfer of basic facts to higher decades up to 100 . It is a wonderful way to show how working with 'ones' or 'units' transfer to working with 'tens'. For example, 3 ones and 3 ones make 6 ones, to 3 tens and 3 tens make 6 tens. It is also an excellent way to demonstrate the concept of regrouping; adding two numbers that make more than 10. By filling the ones column with 10 single cubes it is easy to see that these 10 cubes are now as big as one 10block. This is then exchanged for one whole ten and is moved into the tens compartment.

The Number Track provides opportunities to see the same topic, taught in the Dual Board, in a different light, thus aiding the transfer of conceptual understanding. These materials are part of Stern Kit B, and this level of teaching can be found in Teachers Manual book 3. Further teaching (books 4 and 5) includes multiplication and division, long division, the structure of numbers to $1,000,000$; rounding numbers, fractions, decimal notation, ratio and proportion, the use of percentages and problem solving with these concepts. (Books 4 and 5 availability 2007.)

\section{The evidence}

No formal evaluations of the use of Stern Structural Arithmetic methods and materials with children with Down syndrome have been completed. However, positive reports of successes with their use have been received and a selection follows.

\section{Complex Communication Difficulties Resource Base - Oldham, UK}

Report provided by Fiona Southall, Lead Teacher:

\footnotetext{
"I am using the Stern programme at Counthill Secondary School with 2 pupils:
}

James who is 14 and has Down syndrome is in year 9 - he should be in year 10 but is a year out of cohort. He is working at the higher end of P levels and is completely disapplied from the Key Stage 3 Maths curriculum. We do 35 minutes of Stern 3 times a week. James began in September 06.

"Thomas who is 15, is in year 10 - he should be in year 11 but is a year out of cohort. He has autistic 
spectrum disorder which includes a severe expressive/receptive language disorder ( $1{ }^{\text {st }}$ centile language function) and is working at the low end of level 1 in Maths. He is completely disapplied from the GCSE Maths curriculum and we do 35 minutes of Stern 3 times a week. Thomas began in June 06.

"By November 06 their expressive language had noticeably improved, they are enjoying the role plays and the very small steps approach with repetition means they are secure in one skill before moving onto the next. They both have fine motor difficulties and the size of the equipment is ideal when they are placing cubes and blocks into the apparatus. We did not have a structured scheme to use before and this is ideal. The SENCo is looking at extending it to other pupils with statements. I am also going to borrow the Charlotte Clock from Louise to use with James and Thomas.

"The main strengths of the Stern programme are its structure, visual and concrete cues, use of repetition and the emphasis on developing fine-motor skills. In particular the number formation exercises in the Book 2 Teacher's Manual and Workbook about number formation, have made a noticeable improvement in the legibility of James' written numbers. The sensori-motor exercise suggestions at the end of lessons are also useful. Just doing the workbook exercises in isolation would not be enough for pupils with SEN - it is good that the teacher's manual contains these extra exercises. The teaching (from Book 2) is vital - we use the book constantly as the language is already modelled there - this would be excellent for staff not familiar with the receptive language difficulties associated with the autistic spectrum. We used a wide variety of materials with Thomas before we had Stern, and in hindsight the language we used was too varied and the tasks did not follow on from each other in a structured enough way. The fact that the Stern workbooks and the equipment look the same has made a positive difference

"It is often very difficult to teach children with autism how to count, and we do not think these pupils would have come so far without the blocks and the workbooks. We have worked to the end of Experimenting with Numbers-Book 1 but are revisiting Level 3 to reinforce addition and subtraction. Thomas is ahead of James but the tasks can be done together and differentiated to take account of James' slightly lower ability. A welcome side-effect of the scheme has been the growing friendship and verbal interaction between the participants. Thomas has definitely been producing more speech and what he does say is less echolalic. The boys tease each other (and the teacher!) and love the games where they compete against each other!"

\section{GCSE year pass at Entry Level}

My own daughter Charlotte previously experienced difficulties with Maths. At the age of 10 she had a reading age of 9 and expressive lan- guage, social and behaviour skills were developing nicely. However, her maths age was around 4 years. When Charlotte was 6, I enrolled both of my girls onto the Kumon maths programme, and this worked brilliantly for Emily who was two years older than Charlotte. The early levels - pattern, number recognition and writing numerals certainly helped Charlotte however, when she arrived at the abstract sheets of Add 1 and Add 2 , she ploughed through her homework batches learning methodically. It became clear to both her tutor and me that even with all the practice - sheet after sheet - the concepts were not landing, so my search continued for more materials of a practical nature. Finding Numicon was in my view, the first resource that really made a difference. The plastic number shapes helped her gain some real understanding of the numbers 1 to 10 and because of my work many more children were introduced to the Numicon approach. Charlotte used this programme for well over two years before her interest began to wane. I felt that we needed more variety to maintain interest and to build on what had been achieved. At that time I was aware that Numicon was based on the work of Stern so I went in search of the Stern materials. This took me to New York where I spent a week working with the apparatus and discussed the possibilities of bringing Stern back to the UK. Charlotte worked with Stern for the following three years and in her GCSE year, in collaboration with the Maths teacher at Gillingham school, we worked through the Entry Level course content using Stern. To do so, it meant straddling two Stern programmes leaving gaps in her understanding, however, I was happy with that. Because Charlotte had put so much effort and time into this subject we were keen to give her the opportunity to sit a national exam alongside her peers. The maths teacher Jane Powell reported:

"...We used the Stern programme to support the
Number Strand of the Entry Level Maths Course.
The school worked with Charlotte's mother, pro-
viding copies of the Scheme of Work, sample test
papers and kept her informed of the topics being
covered. Charlotte was then able to continue her
work at home. Using the Stern equipment: the
Number Track, Dual Board and 20-Tray provided
both visual and kinaesthetic learning experiences.
The short term targets and frequent repetition of
Stern's programme was also ideally suited to Char-
lotte's style of learning. We learned a great deal
from using this programme. In particular, maths
language - we felt that there is a need to begin
linking the language of the National Curriculum
at a much earlier stage because this left Charlotte
at a disadvantage in the public exam. Neverthe-
less, Stern was a significant factor in Charlotte's
success in gaining the Entry Level qualification."

\section{$P$ scales}

The $P$ scales are a set of indicators for recording the achievement of pupils with special educational needs who are working towards the first level of the National Curriculum.

\section{National Curriculum}

The statutory teaching curriculum in England for pupils up to the age of 16. It determines the content of what will be taught and sets attainment targets for learning.

National Curriculum Key Stages and levels

The English National Curriculum Key Stage 1 is for pupils aged 5-7 years, Key Stage 2 for 7-11 years, Key Stage 3 for 11-14 years and Key Stage 4 for students aged 14-16 years. The programmes of study also map out a scale of attainment within the subject. In most Key Stage 1, 2, and 3 subjects, these attainment targets are split into eight levels. By the end of Key Stage 1 (aged 7), most typically developing children will have reached level 2 , and by the end of Key Stage 2 (aged 11), most will be at level 4 .

\section{GCSE}

General Certificate of Secondary Education, graded $A^{*}$ to $G$, the subject based examinations pupils normally take at the end of Key Stage 4, age 16.

\section{Entry Level certificates} Accredited qualifications available to schools for pupils working below the level of GCSE. Entry level 1,2 and 3 are broadly equivalent to National Curriculum level 1,2 and 3. Entry levels may cover one, two or three of these levels. 


\section{Two Years Using Stern Materials for George}

Report provided by Jennifer Senn, Learning Support Assistant:

George has Down syndrome and is 8 years old. He attends The Russell School, in Petersham, Richmond-upon-Thames. George has been using Stern for over 2 years and is working at level II. It has helped him understand many things. The Teachers manual is clear and easy to use albeit there is a lot in there to read and do! The activities are easy to deliver and the repetition is constant and ongoing: George now has a target to learn doubles and bonds to 20. George has learnt: • How to follow instructions, take turns - To recognise each block by size/colour - One more one less, before after, what comes next • Making number lines, finding missing numbers $\cdot$ Number bonds to $10 \cdot$ Estimating $\cdot$ How many altogether $\cdot$ Doubles $1+1,2+2,3+3,4+4,5+5$, halves $\cdot$ Adding one more • Odd and even numbers

\section{Relationship to UK Revised Primary Mathematics Framework}

A major change in focus in the renewed Primary Mathematics Framework, published in Autumn 2006, has been an increased emphasis on calculation and its prerequisites in terms of understanding the number system and knowing number facts. The expectations for a quick ability at calculation and the recall of number facts have been increased, and age related expectations for learning times tables have been brought forward earlier than hitherto. Three of the seven strands laid out in the Framework deal with the increased focus on calculation:-

- Counting and understanding number counting, comparing and ordering numbers and describing relationships between them

- Knowing and using number facts - having secure knowledge of number facts which can be recalled quickly and used and applied appropriately, together with a good understanding of the four operations

- Calculating - being able to calculate efficiently and accurately, mentally

Of course, these three strands are central to the others, which include using and applying mathematics as well as problem solving.

The Stern Structural Arithmetic Apparatus is based on psychological insights into how children learn mathematics concepts and processes best, and can be used to allow plenty of independent practice by children in order to internalise the number bonds for the essential quick recall. The programme stresses comparison, ordering and identifying and describing relationships, as required by the Framework, and provides a multi-sensory approach which again aids the recall process. It is also, thorough in helping children to understand about "undoing what has been done", i.e. about the reversibility of processes, another important point in the renewed Framework.

In terms of the basic structure of learning numeracy the sequence of acquisition suggested in the Stern programme is very similar to that of the renewed Framework. The linear development of the central structure of the number system follows the same logical pathway, moving from understanding both cardinal and ordinal values of numbers to ten, addition and subtraction facts of numbers to ten, then of the teens and twenties, and after that of place value and two, three and four digit numbers. Teaching units, games, and activities offering multiple opportunities for repeated practice and reinforcement are of great value in developing the accurate storage of the necessary number facts.

Received: 7 February 2007; Accepted 14 February 2007; Published online: 30 July 2007.

\section{BOX $1 \mid$ Materials required and costs}

Kit A - comprises full range of apparatus to teach basic facts to 10 and includes Teachers Manual. Use: Nursery, at home from age 3 , Reception class \& SEN $£ 98.70+p \& p$ + VAT

Book 2 Series - Teachers Manual $£ 26.00$, Pupil's Workbooks $£ 2.00$ each + p\&p. Zero VAT.

Kit B - Apparatus only $£ 120.00+p \& p+$ VAT Book 3 series - Teachers Manual $£ 26.00$, Pupil's Workbooks $£ 2.00$ each + p\&p zero VAT

Book 4 series - Teachers Manual $£ 30.00$, Teachers Photocopiable Resource $£ 26.00+$ p\&p zero VAT
Book 5 series - Teachers Manual $£ 30.00$, Teachers Photocopiable Resource $£ 26.00$ + p\&p zero VAT

Teaching Series 3, 4, and 5 are taught with Kit B apparatus.

What to Purchase

Parents - from age 3 onwards would use Kit $A$. If your child is ready to learn to write the numerals 1 to 10 , Book 2 and accompanying pupils workbook would be required. To progress to the bonds to 20 and 2-place numbers Kit $B$ is required together with the Book 3 series. Subsequent teaching requires the addition of books 4 and then books 5 .
Early Years Settings - will require Kit A only.

Primary Settings - will require Kit A initially, adding Teaching Manuals Book 2 series for consolidation and written work practise. Further additions are Kit B, and Teaching Manuals Book 3 series as pupils progress.

Secondary Settings - Kit B only as this can be adapted for those students who are still working with the numbers 1 to 10 and Book 3 series. If adapting Kit B to teach basic facts to 10 , then appropriate Teaching Manuals will be required. 